\title{
Link Resource Adaptation for Multiantenna Bit-Interleaved Coded Multicarrier Systems
}

\author{
Ivan Stupia, Vincenzo Lottici, Filippo Giannetti, and Luc Vandendorpe, Fellow, IEEE
}

\begin{abstract}
The availability of flexible radio interfaces capable of adapting their configuration to the time-varying operating environment is the key response to the demand encountered in modern wireless networks for high data rates under strict quality of service $(\mathrm{QoS})$ constraints. To this end, this paper develops a novel link resource adaptation (LRA) scheme for soft-decoded multiantenna (MIMO) bit interleaved coded orthogonal frequency division multiplexing (BIC-OFDM) transmissions employing automatic repeat request (ARQ) mechanisms. As the first step, a simple link performance evaluation model based on the effective signal-to-noise ratio (SNR) mapping concept is derived in a closed-form expression that it is shown to yield better accuracy than previous techniques. Then, an effective LRA strategy is formulated taking advantage of that framework. The aim is maximizing the goodput (GP) metric, that is to say, the number of information bits delivered without error to the user by unit of time, over the available radio resources, such as the power distribution on the subchannels, coding rate, modulation order and the MIMO configuration. The numerical results demonstrate considerable performance gains compared with nonadaptive transmissions, while keeping the computational complexity at affordable levels in view of the specific structure of the GP objective function.
\end{abstract}

Index Terms-Automatic repeat request (ARQ), bit-interleaved coded modulation, cross-layer link resource adaptation, goodput, multiple-input multiple-output (MIMO), orthogonal frequency division multiplexing (OFDM), power allocation.

\section{INTRODUCTION}

$\mathbf{N}$ EXT-GENERATION multimedia services over seamless and pervasive wireless networks call for high data rates under strict quality of service (QoS) constraints [1]. The harsh multipath propagation conditions typically experienced in both urban outdoor and indoor scenarios along with the scarcity of available bandwidth, however, make the design of reliable

Manuscript received October 05, 2010; revised July 28, 2011 and January 05, 2012; accepted March 04, 2012. Date of publication April 03, 2012; date of current version June 12, 2012. The associate editor coordinating the review of this manuscript and approving it for publication was Prof. Shahram Shahbazpanahi. This work was supported by the European Commission in the Framework of the FP7 Network of Excellence in Wireless COMmunications NEWCOM++ (Contract 216715). This paper was presented in part at the 7th International Workshop on Multi-Carrier Systems \& Solutions (MC-SS), Herrsching, Germany, May 2009, and in part at the IEEE International Conference on Communications (ICC), Dresden, Germany, June 2009.

V. Lottici and F. Giannetti are with the Department of Information Engineering, University of Pisa, I-56122 Pisa, Italy (e-mail: vincenzo.lottici@iet. unipi.it; filippo.giannetti@iet.unipi.it).

I. Stupia and L. Vandendorpe are with the Université Catholique de Louvain, B-1348 Louvain-la-Neuve, Belgium (e-mail: ivan.stupia@uclouvain.be; luc.vandendorpe@uclouvain.be).

Color versions of one or more of the figures in this paper are available online at http://ieeexplore.ieee.org.

Digital Object Identifier 10.1109/TSP.2012.2192110 and spectrally efficient packet data transmission systems a particularly demanding task. To this end, a number of efficient solutions have been proposed, like multicarrier (MC) modulation, in the form of orthogonal frequency division multiplexing (OFDM) [2], advanced channel coding as the bit interleaved coded modulation (BICM) [3], multiple-input multiple-output (MIMO) communication [4]-[7], and automatic repeat request (ARQ) mechanisms [8]. A further boosting of the reliability and efficiency figures resides in thinking of flexible radio interfaces capable of adjusting their transmission parameters on the fly according to the time-varying conditions of the operating environment, what is known as link resource adaptation (LRA); see [9] and references therein. Hence, combining the LRA concept with the above techniques working at both the physical and data link layers, the link configuration parameters, including data rate, transmit power, coding rate or scheme, modulation order and MIMO scheme, can be adaptively and jointly optimized in a cross-layer oriented approach [10].

Relation to Prior Work: Among the various LRA techniques recently appeared in the literature, one of the most recognized criterion is based on the maximization of the mutual information [11]. Based on such a criterion, bit and power allocation strategies have been proposed for BIC-OFDM and MIMO-BICM systems in [12] and [13], respectively. When one has to do with more practical modulation and coding schemes, however, alternative approaches different from those in [11]-[13] could be more convenient. Moreover, the presence of the ARQ retransmission mechanism at the data link layer should be taken into account as well in order to quantify the trade-off between data rate and link reliability. Towards this goal, a significant figure of metric has thus been detected in the offered layer 3 data rate, or goodput (GP) for short. In this context, [10] and [14] adopt the GP as performance metric to select the best combination of modulation and coding rate with uniform bit and power loading. In [15], instead, the GP criterion motivates the problem of optimally distributing bits and power across a set of subchannels in a coded adaptive OFDM system with hard Viterbi decoding. In particular, the hard decisions on the coded binary symbols are exploited so as to split the cross-layer nature of the problem by using the uncoded BER (at the decoder input) as an intermediate performance metric. On the other side, when soft Viterbi decoding is considered this layer separation is not trivial at all. Thus, to make feasible the LRA multiparametric optimization problem (OP), the derivation of an accurate link performance metric providing a compact and manageable analytic representation of the GP performance is a crucial task. In this sense, one of the most promising link performance evaluation (LPE) method to be mentioned is the effective signal-to-noise 
ratio (SNR) mapping (ESM) [16], such as exponential ESM (EESM), logarithmic ESM (LESM), capacity ESM (CESM), and mutual information ESM (MIESM) [17]. In particular, the EESM has the main advantage of providing a closed-form mapping function, but unfortunately, its generalization to high order modulations [18] does not exist. Differently from EESM, the MIESM solution includes two separates models, one for modulation and the other for coding, thereby enabling the separation between layers and making LRA very convenient [19], although the mutual information mapping function has not a closed-form expression.

Rationale of the Proposed Approach and Contribution: In this paper, we propose a novel LRA strategy aimed at maximizing the GP performance for a MIMO BIC-OFDM system with soft Viterbi decoding. This goal is pursued through the specific approach briefly outlined hereafter.

- Assuming that the real future channel propagation conditions cannot be predicted, in the formulation of our LRA problem we replace the GP metric of interest with an approximation given by the expected goodput (EGP) [14]. This is analytically derived upon a novel LPE method belonging to the broader class of ESM methods, and just plays the role of prediction metric about the the actual link GP performance.

- The OP having the EGP as objective function is optimally and analytically solved under the constraint of limited transmit power.

- Extensive time-domain numerical simulations of the overall system, featuring coded multicarrier signal generation, multipath propagation, data detection and ARQ retransmission mechanism, are carried out to corroborate the effectiveness of the proposed LRA method for various system configurations in a "real-world" scenario, by quantifying the actual GP performance and not the approximated EGP metric.

Several features differentiate the proposed LRA from previous works and define our contribution.

- A novel LPE tagged as cumulant generating function based ESM, or $\kappa \mathrm{ESM}$ for short [20], which combines together the simplicity of the EESM with the accuracy of MIESM, enables a simple formulation of the multiparametric OP.

- In light of the particular $\kappa \mathrm{ESM}$ methodology, the structure of the GP metric is exploited in order to split the constrained OP into two subproblems, thus keeping the computational complexity at affordable levels.

- A power allocation (PA) algorithm is presented which aims at maximizing the GP metric as a function of the link configuration parameters, under the assumption of ideally known channel state information (CSI), called as goodputoriented power allocation, or GOPA for short.

- Taking advantage of the separation between modulation and coding models in the $\kappa \mathrm{ESM}$, the maximization of the EGP objective function allows to solve the multiparametric $\mathrm{OP}$ in the domain of power distribution across subchannels, coding rate, modulation order and MIMO configuration.

Organization: The rest of the paper is organized as follows. We start on describing in Section II the MIMO BIC-OFDM system we adopt as reference model. After reviewing the background of the ESM method, Section III defines the EGP objective function based on the $\kappa \mathrm{ESM}$ model. Section IV first formalizes the solution of the constrained optimization GOPA problem, and then, applies it to the proposed LRA algorithm. Finally, Section V is devoted to evaluating performance via computer simulations over typical wireless channels, followed in Section VI by some concluding remarks.

Notations: Matrices are in upper case bold while column vectors are in lower case bold, $(\cdot)^{T}$ denotes transpose, $(\cdot)^{H}$ denotes conjugate transpose, $[\boldsymbol{A}]_{i, j}$ denotes the $(i, j)$ entry of the matrix $\boldsymbol{A}, \operatorname{diag}(\cdot)$ converts the main diagonal of an $N \times N$ matrix into an $N \times 1$ vector, $\mathbf{1}_{N}$ is the $N \times 1$ vector with all components one, $\delta_{n}$ is the Kronecker function taking the value 1 when $n=0$ and zero otherwise, and $\mathrm{E}\{\cdot\}$ denotes statistical expectation.

\section{System Model Overview}

The block diagram of the MIMO BIC-OFDM communication system we are dealing with is schematized in Fig. 1. In this framework, we take a packet (usually corresponding to an IP packet or a layer 3 control signalling message) as the elementary piece of information to be transmitted over the radio interface and delivered error-free to the end-user. In view of this packet-centric paradigm [1], the overall radio-access processing scheme can be split into the packet-processing and frame-processing components. Packet-processing consists in the combination of ARQ and channel coding mechanisms. At the transmitter side, each packet queued within a buffer is one-to-one mapped to a radio link control (RLC) sublayer protocol data unit (PDU), which includes the header, the corresponding payload received from upper layers and a cyclic redundancy check (CRC) coded sequence for error detection. The packets decoded at the receiver without any errors are properly stored for the delivery to the end-user, whereas for the ones received with errors a retransmission is requested via a feedback channel. The transmitter is aware of either conditions upon getting from the receiver a positive or negative acknowledgement, denoted respectively as ACK or NACK for short. The next step in the packetprocessing flow at the transmitter is to map one RLC PDU to exactly one FEC block, with the result that each packet is self-decodable. Specifically, the RLC PDU consisting of $N_{u}=N_{h}+$ $N_{p}+N_{\text {CRC }}$ binary information symbols, where $N_{h}, N_{p}$ and $N_{\text {CRC }}$ are the sizes of the header, payload and CRC sections, respectively, is encoded with rate $r$. The coded sequence having size $N_{b}=\frac{N_{u}}{r}$ is accepted by the frame processing section to perform the basic physical layer operations of modulation and symbol mapping to the available physical resources. In the sequel, we refer to the sequence of modulation symbols conveying each coded sequence as a frame. In details, the encoded binary symbols $\left\{b_{j}\right\}, 1 \leq j \leq N_{b}$, corresponding to each RLC PDU are randomly interleaved according to the BICM model and mapped to the sequence $\left\{a_{l, n, q, i}\right\}$. The index $l$, with $1 \leq l \leq L$, defines the position within the transmitted frame made of $L$ consecutive OFDM symbols each with length $T_{\mathrm{B}}, n$, with $1 \leq n \leq N$, is the index out of the overall $N$ subcarriers, $q$, with $1 \leq q \leq M$, is the index of the spatial stream corresponding to the $n$th subcarrier, and $i$, with $1 \leq i \leq m^{(n, q)}$, is the position of the coded binary symbol within the label of 


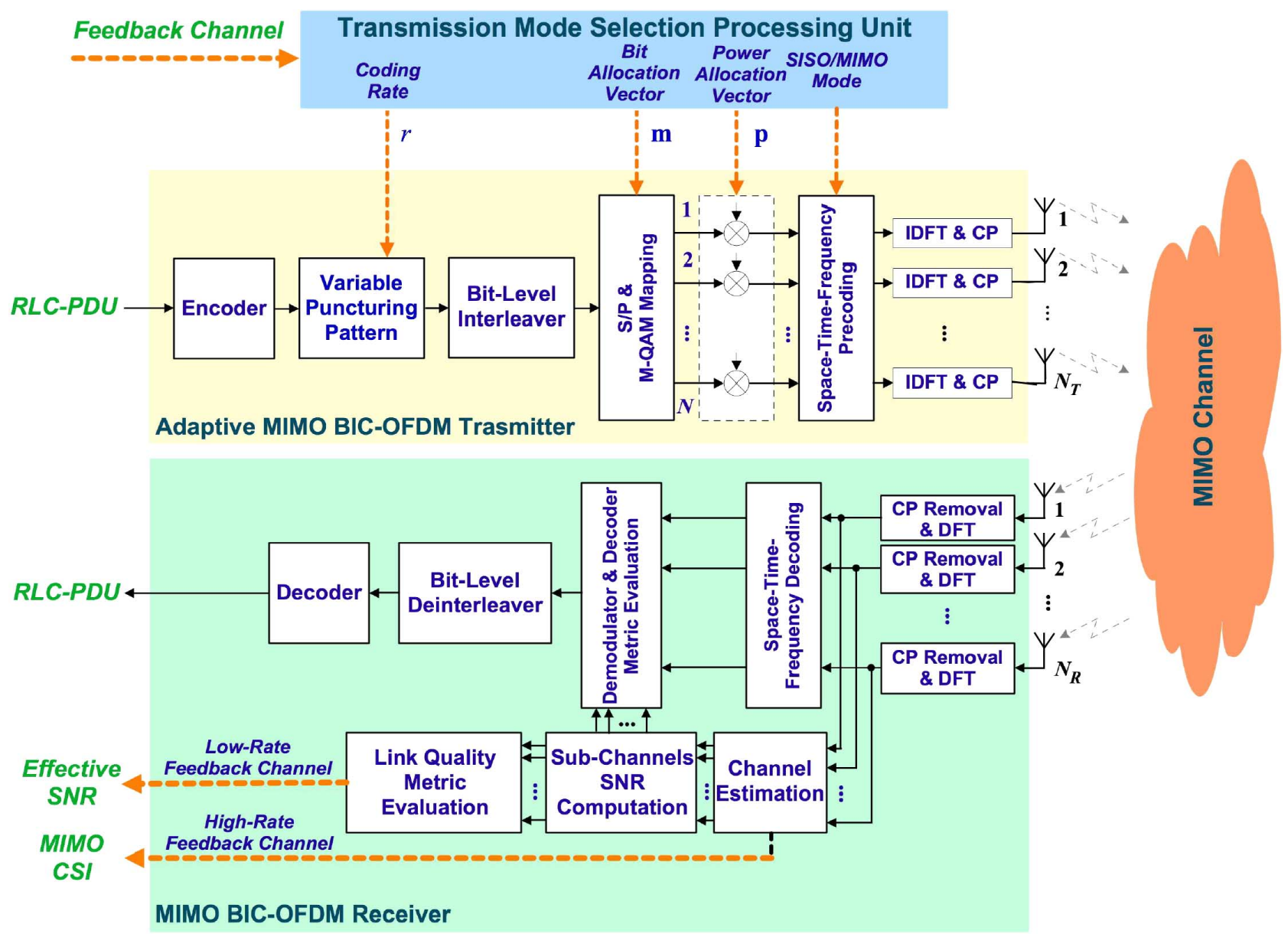

Fig. 1. Block diagram of the MIMO BIC-OFDM system.

the modulation symbol across the $n$th subcarrier and $q$ th spatial stream. The subcarrier $n$ and the spatial stream $q$ represent the subchannel $(n, q) \in \mathcal{C}$, where the $\operatorname{set} \mathcal{C} \triangleq\{(n, q) \mid, 1 \leq n \leq$ $N, 1 \leq q \leq M\}$ contains $C \triangleq N \cdot M$ index pairs of the active subchannels.

The coded and interleaved sequence is partitioned into binary $m^{(n: q)}$-tuples, each of which is Gray-mapped onto the complex-valued symbol $s_{l}^{(n, q)} \in \chi^{(n, q)}$, where $\chi^{(n, q)}$ is a unitary-energy QAM constellation. The symbol vector $\boldsymbol{s}_{l}^{(n)} \triangleq\left[s_{l}^{(n, 1)}, \cdots, s_{l}^{(n, M)}\right]^{T}$ is then multiplied element-by-element by the vector $\boldsymbol{a}^{(n)} \triangleq \sqrt{S}\left[\sqrt{p^{(n, 1)}}, \cdots,{\sqrt{p^{(n, M)}}}^{T}\right.$, where $S$ is the average power which can be transmitted on any subchannel, while $p^{(n, q)} \geq 0$ represents the normalized power which is actually allocated on subchannel $(n, q)$ according to the constraint $\mathbf{1}_{C}^{T} \boldsymbol{p} \leq P$, with $\boldsymbol{p} \triangleq\left[\boldsymbol{p}^{(1)^{T}}, \cdots, \boldsymbol{p}^{(N)^{T}}\right]^{T}$ and $\boldsymbol{p}^{(n)} \triangleq\left[p^{(n, 1)}, \cdots, p^{(n, M)}\right]^{T}$, with $P$ being the maximum available power at the transmitter side.

The scaled symbol vector is then space-time (ST) encoded in accordance with a MIMO scheme based on $N_{\mathrm{T}}$ transmit and $N_{\mathrm{R}}$ receive antennas, and the result for each antenna is frequency-mapped onto the $N$ available subcarriers. The resulting OFDM signals are transmitted over a MIMO frequency-selective fading channel, assumed to be time-invariant within the frame interval, but randomly independent across consecutive frames. At the receiver, the signal is ST decoded, soft demapped, deinterleaved and eventually decoded. In line with the system model outlined above, the normalized output of the ST decoder can be expressed as

$$
\boldsymbol{y}_{l}=\boldsymbol{\Upsilon} \boldsymbol{s}_{l}+\boldsymbol{\nu}_{l}
$$

where $\boldsymbol{s}_{l} \triangleq\left[\boldsymbol{s}_{l}^{(1)^{T}}, \cdots, \boldsymbol{s}_{l}^{\left(N^{T}{ }^{T}\right.}\right]^{T}, \boldsymbol{\nu}_{l}$ is a $C$-dimensional noise vector whose entries are circularly symmetric complex-valued Gaussian RVs with zero mean and unit variance, and $\Upsilon$ is a $C \times C$ diagonal matrix whose generic entry expressed by $[\Upsilon]_{(n-1) M+q,(n-1) M+q} \triangleq \Upsilon^{(n, q)} \triangleq \frac{\sqrt{S} \sqrt{p^{(n, q)}} A^{(n, q)}}{\sigma^{(n, q)}}$ collects the square-roots of the postprocessing SNRs for the subchannel $(n, q)$, which depends on the complex-valued multipath channel coefficient $A^{(n, q)}$ and $\sigma_{w}^{(n, q)}$ including both the thermal noise component and the multistream interference of the particular MIMO scheme. For the sake of simplicity, the corresponding postprocessing SNRs can be arranged into the $C$-sized vector $\boldsymbol{\gamma} \triangleq \operatorname{diag}\left\{\boldsymbol{\Upsilon} \boldsymbol{\Upsilon}^{H}\right\}$, whose entries are $[\gamma]_{(n-1) M+q} \triangleq \gamma^{(n, q)} \triangleq\left[\Upsilon^{(n, q)}\right]^{2}$.

\section{LinK Performance Evaluation}

In this section, we put our focus on an improved LPE model designated as $\kappa \mathrm{ESM}$, that will play a key role in the maximization of the GP metric, as detailed in Section IV. 


\section{A. The ESM Concept}

The idea behind the ESM concept consists in mapping the instantaneous SNRs measured on the receiver subchannels into a scalar SNR that would yield the same performance in an "equivalent" coded system operating over an AWGN channel. Stated formally, for a given transmission mode (TM) defined as the collection of modulation size, coding rate and MIMO scheme, the $C$ SNR values included in $\boldsymbol{\gamma}$ are compressed into the scalar effective SNR $\gamma_{\text {eff }}$ so that the condition

$$
\operatorname{PER}_{\text {AWGN }}\left(\gamma_{\text {eff }}\right)=\operatorname{PER}_{\text {Fad }}(\boldsymbol{\gamma})
$$

holds, where $\mathrm{PER}_{\mathrm{AWGN}}$ and $\mathrm{PER}_{\mathrm{Fad}}$ refer to the AWGN equivalent system and the actual one affected by fading, respectively. In equivalent words, this means that the ESM reduces the LPE problem to: 1) evaluate $\boldsymbol{\gamma}$;2) compress $\boldsymbol{\gamma}$ into $\gamma_{\text {eff }}$ through (2); and 3) evaluate at the SNR value $\gamma_{\text {eff }}$ the PER curve obtained analytically or by simulations for the equivalent AWGN coded transmission. Hence, depending on the rule adopted to translate the SNR vector $\gamma$ into the scalar $\gamma_{\text {eff }}$, a number of ESM methods may come up with different accuracy-versus-complexity tradeoff levels. In the next subsection, we propose a novel ESM model based on the cumulant generating function (CGF) of the log-likelihood ratios (LLRs) metric employed by the BICM soft decoder.

\section{B. Definition of $\kappa E S M$}

The $\kappa$ ESM concept is based on the in-depth evaluation of the pairwise error probability (PEP) figure. To identify the essentials of this approach, consider a reference codeword $\boldsymbol{b} \triangleq\left[b_{1}, \cdots, b_{d}\right]^{T}$ and the competitor codeword $\boldsymbol{b}^{\prime} \triangleq\left[b_{1}^{\prime}, \cdots, b_{d}^{\prime}\right]^{T}$, both originating from the same state of the trellis code and merging after $d$ steps. Denote with $\hat{\boldsymbol{b}}$ the corresponding decoder output, then focus on the calculation of the PEP between $\boldsymbol{b}$ and $\boldsymbol{b}^{\prime}$, namely $\operatorname{PEP}(d) \triangleq \operatorname{Pr}\left\{\hat{\boldsymbol{b}}=\boldsymbol{b}^{\prime} \mid \boldsymbol{b}, \boldsymbol{\Upsilon}\right\}$, where $\Upsilon$ is the diagonal matrix defined in (1). Since the underlying rationale is to exploit the inherent behavior of BICM as an equivalent "binary" modulation, we first review in the sequel two basic approximations originally proposed in [21] which support such a description, and then apply both of them to the case of interest.

1) The Gaussian Approximation. In [21], it is shown that in the limit of large SNR: 1) the tail of the distribution of the LLRs is well approximated by a Gaussian shape; and 2) the bit error rate (BER) decays exponentially with SNR. Then, we observe that in our LRA problem we are interested about the event of successfully receiving the entire packet without any error. Thus, under the assumption of sufficiently large packet size (as in our case), this safely means that the BER gets low-to-medium values, just the region where it has exponential decay and the Gaussian approximation holds tight. As a consequence, assuming ideal random interleaving and that the set of active subchannels can be schematized as a memoryless binary-input output-symmetric (BIOS) channel, the PEP can be computed as the tail probability

$$
\operatorname{PEP}(d) \simeq \mathrm{Q}\left(\sqrt{-2 d \kappa_{\Lambda}(\hat{u})}\right)
$$

where $\mathrm{Q}(\cdot)$ is the tail probability of the standard normal distribution, $\kappa_{\Lambda}(u) \triangleq \log \mathcal{M}_{\Lambda}(u)$ is the CGF and $\mathcal{M}_{\Lambda}(u) \triangleq \mathrm{E}\left\{e^{u \Lambda}\right\}, u \in \mathbb{C}$, is the moment generating function (MGF) of the RV specified by the LLR metric

$$
\Lambda_{j} \triangleq \log \frac{\sum_{\tilde{s} \in \chi_{b_{j}^{\prime}, i}^{(n, q)}} \exp \left[-\left|y_{l}^{(n, q)}-\Upsilon^{(n, q)} \tilde{s}\right|^{2}\right]}{\sum_{\tilde{s} \in \chi_{b_{j}, i}^{(n, q)}} \exp \left[-\left|y_{l}^{(n, q)}-\Upsilon^{(n, q)} \tilde{s}\right|^{2}\right]}
$$

for the $j$ th coded symbol, $1 \leq j \leq N_{b}$, and $\hat{u}$ is the value (saddlepoint) so that $\kappa_{\Lambda}^{\prime}(\hat{u})=0$ which is placed at $\hat{u}=\frac{1}{2}$. In (4), $\chi_{b, i}^{(n, q)}$ is the subset of the symbols belonging to the QAM constellation $\chi^{(n, q)}$ adopted on the $(n, q)$-subchannel whose $i$ th label bit is equal to $b$. Further, note that the dependence of the indexes $l$ (block), $n$ (subcarrier), $q$ (spatial stream) and $i$ (position within the symbol label) on the index $j$ (coded symbol) has been dropped for the sake of simplicity.

2) The High-SNR Approximation. With the assumption of sufficiently large SNR and by resorting to the dominated convergence theorem (DCT), it is demonstrated in [21] that the MGF is dominated by the term relevant to the symbol $\bar{s}$ closest to $s_{l}^{(n, q)}$ in the complementary subset $\chi_{b_{j}^{\prime}, i}^{(n, q)}$, thereby obtaining

$$
\mathcal{M}_{\Lambda}(u) \simeq \mathrm{E}\left\{\exp \left[-\gamma^{(n, q)} d^{2}\left(s_{l}^{(n, q)}, \bar{s}\right)\left(u-u^{2}\right)\right]\right\}
$$

where $d\left(s, s^{\prime}\right)$ is the Euclidean distance between the complex-valued symbols $s$ and $s^{\prime}$, and the expectation is taken over all the nuisance parameters, i.e., the noise, the modulation symbol $s_{l}^{(n, q)}$ and the position of the coded symbol within the label of the QAM symbols in the complementary subset.

It is worth remarking here that the applicability of the two approximations introduced above will be testified in Section V by showing that, at each $\frac{E_{s}}{N_{0}}$ of practical interest and regardless of the adopted setup, the proposed LRA scheme always selects the best-performing TM thus matching the link parameters to the instantaneous channel conditions when adopting different coding scheme and modulation orders.

To compute the expectation in (5), let us bear in mind the following remarks: 1) due to the Gray mapping rule, we have $d\left(s_{l}^{(n, q)}, \bar{s}\right)=\mu d_{\min }^{(n, q)}$, where $\mu$ is a positive integer and $d_{\min }^{(n, q)}$ is the minimum Euclidean distance between the symbols of the constellation adopted on the $(n, q)$-subchannel; 2) each of the $m^{(n, q)}$ label bits conveyed by the $(n, q)$-subchannel has $2^{m^{(n, q)}-1}$ symbols on its complementary subset, so that the total number of terms to be averaged results $m^{(n, q)} \cdot 2^{m^{(n, q)}-1} ; 3$ ) the distance $d\left(s_{l}^{(n, q)}, \bar{s}\right)$ takes $\frac{\sqrt{2^{m^{(n, q)}}}}{2}$ distinct values so that $1 \leq \mu \leq \frac{\sqrt{2^{m^{(n, q)}}}}{2}$; 4) by defining the number of symbols at distance $\mu d_{\text {min }}^{(n, q)}$ from the nearest neighbor in the complementary subset as $\psi^{(n, q)}(\mu)$, we get $\sum_{\mu=1}^{\frac{\sqrt{2^{m^{(n, q)}}}}{{ }^{2}}} \psi^{(n, q)}(\mu)=$ $m^{(n, q)} \cdot 2^{m^{(n, q)}-1} \forall(n, q) \in \mathcal{C}$, where $\psi_{4-\mathrm{QAM}}^{(n, q)}(\mu)=4 \delta_{\mu-1}$, $\psi_{16-\mathrm{QAM}}^{(n, q)}(\mu)=24 \delta_{\mu-1}+8 \delta_{\mu-2}, \psi_{64-\mathrm{QAM}}^{(n, q)}(\mu)=112 \delta_{\mu-1}+$ 
$48 \delta_{\mu-2}+16 \delta_{\mu-3}+16 \delta_{\mu-4}$ for the 4-, 16- and 64-QAM formats, respectively; and 5) the probability that the generic coded symbol is sent through the $(n, q)$-subchannel is $\frac{m^{(n, q)}}{\sum_{(i, j) \in \mathcal{C}} m^{(i, j)}}$. Therefore, collecting the above results together, the MGF in (5) turns out to be

$$
\begin{aligned}
& \mathcal{M}_{\Lambda}(u) \simeq \frac{1}{\sum_{(i, j) \in \mathcal{C}} m^{(i, j)}} \\
& \cdot \sum_{(n, q) \in \mathcal{C}} \frac{\sqrt{2^{m^{(n, q)}}}}{\sum_{\mu=1}^{2}} \frac{\psi^{(n, q)}(\mu)}{2^{m^{(n, q)}-1}} e^{-\gamma^{(n, q)}\left[\mu d_{\text {min }}^{(n, q)}\right]^{2}\left(u-u^{2}\right)}
\end{aligned}
$$

and accordingly, the CGF actually required in (3) to evaluate the PEP for two codewords differing in $d$ bits results as $\kappa_{\Lambda}(\hat{u})=$ $\log \mathcal{M}_{\Lambda}(\hat{u})$. Hence, considering that: 1$)$ the PER depends on the $\operatorname{PEP}(d)$ function; and 2) (3) can be viewed as the PEP of an equivalent binary system operating over an AWGN channel with SNR equal to $-\kappa_{\Lambda}(\hat{u})$, we obtain the main result of this section, namely the definition of the $\kappa$ ESM performance model, as stated in the following proposition.

Proposition 1: The $\kappa \mathrm{ESM}$ consists of the modulation model

$$
\Omega^{(n, q)} \triangleq \sum_{\mu=1}^{\frac{\sqrt{2^{(m, n q)}}}{2}} \frac{\psi^{(n, q)}(\mu)}{2^{m^{(n, q)}-1}} e^{-\gamma^{(n, q)}\left[\mu d_{\min }^{(n, q)}\right]^{2} / 4 \beta_{\kappa \mathrm{ESM}}}
$$

where the weighted sum of exponential functions are computed for each $(n, q)$-subchannel, which experiences the SNR level $\gamma^{(n, q)}$ and conveys (for a given modulation order) $m^{(n, q)}$ label bits, with $(n, q) \in \mathcal{C}, \beta_{\kappa \mathrm{ESM}}$ being a tuning factor dependent only on the coding scheme. The quantities $\Omega^{(n, q)}$ are then accumulated in the coding model and normalized to provide the scalar effective SNR

$$
\gamma_{\kappa \mathrm{ESM}} \triangleq-\beta_{\kappa \mathrm{ESM}} \log \left\{\frac{1}{\sum_{(i, j) \in \mathcal{C}} m^{(i, j)}} \sum_{(n, q) \in \mathcal{C}} \Omega^{(n, q)}\right\} .
$$

As final step, the predicted link quality is obtained by reading the PER curve of the equivalent binary coded system operating over AWGN from a look-up table (obtained by either simulations or using a tight upper bound) at the SNR value corresponding to $\gamma_{\kappa \text { ESM }}$.

It is now worth emphasizing the original features which differentiate the $\kappa \mathrm{ESM}$ model with respect to other techniques available in the literature:

1) it enables PER prediction at the transmitter side based only on the scalar effective SNR (8) instead of the whole CSI;

2 ) it separates the modulation and coding models, thus enabling the split between layers and making the cross-layer OP easy to manage;

3 ) it allows the use (required for instance for the turbo codes) of a tuning factor $\beta_{\kappa \mathrm{ESM}}$ which is independent of the modulation format adopted on each subcarrier;

4) it provides a mapping function for the modulation model which can be expressed in a simple closed-form.

\section{Definition of the Expected Goodput Objective Function}

One of the main outcomes of the $\kappa \mathrm{ESM}$ framework developed in Section III-B is the formulation of a simple link quality metric that can take equally into account the presence of both error-correction and packet-retransmission mechanisms.

Let us start with thinking about the standard GP metric defined as the ratio between the number of error-free payload bits and the average transmission time spent on the attempt of successfully delivering them. In order to reach a tractable formulation of the objective function, we assume that each transmitted packet experiences the same (currently known) channel conditions within its (possible) ARQ retransmissions. That way, complex channel prediction techniques providing side information such as estimates of delay-spread, Doppler bandwidth, and so on, are no longer required. This assumption leads to the concept of expected goodput (EGP), as originally proposed in [14]. Notice that, although the EGP resembles the actual GP metric only if the channel stays invariant or is highly correlated within consecutive retransmission intervals, nevertheless it is advantageously possible to obtain a simple objective function of convex type, which enables a considerable improvement of the actual GP link performance. It is worth of being pointed out, however, that in all of the simulation tests illustrated in Section V, the assumption about the time-invariance of the channel will be dropped, so as to adhere to more realistic propagation environments.

Coming back to the analytical derivation of the objective function, we observe first that the probability of a successful RLC-PDU delivery within the retry limit $R$ can be written as

$$
\lambda_{\mathrm{s}}(\boldsymbol{\varphi}, \boldsymbol{p})=1-\left\{\operatorname{PER}_{\boldsymbol{\varphi}}\left[\gamma_{\kappa \mathrm{ESM}}(\boldsymbol{\varphi}, \boldsymbol{p})\right]\right\}^{R+1}
$$

where $\boldsymbol{p}$ represents the $C$-dimensional vector carrying the power coefficients allocated on the subchannels, $\varphi$ represents a generic $\mathrm{TM}, \gamma_{\kappa \mathrm{ESM}}(\boldsymbol{\varphi}, \boldsymbol{p})$ is the $\kappa \mathrm{ESM}$ effective SNR (8) compressing the current CSI and thus depending on both $\varphi$ and $p$, while the function which maps $\gamma_{\kappa \mathrm{ESM}}$ into the PER value depends on the $\varphi$ alone. Hence, the expected average transmission intervals for successful and failed packet delivery result, respectively, as

$$
\begin{aligned}
& \mathcal{T}_{\mathrm{s}}(\boldsymbol{\varphi}, \boldsymbol{p})=\left\{1-\operatorname{PER}_{\boldsymbol{\varphi}}\left[\gamma_{\kappa \mathrm{ESM}}(\boldsymbol{\varphi}, \boldsymbol{p})\right]\right\} \\
& \cdot \sum_{h=0}^{R} \frac{\left\{\operatorname{PER}_{\boldsymbol{\varphi}}\left[\gamma_{\kappa \mathrm{ESM}}(\boldsymbol{\varphi}, \boldsymbol{p})\right]\right\}^{h}}{\lambda_{\mathrm{s}}(\boldsymbol{\varphi}, \boldsymbol{p})}(h+1) T_{\mathrm{RLC}}(\boldsymbol{\varphi}) \\
& \mathcal{T}_{\mathrm{f}}(\boldsymbol{\varphi})=(R+1) T_{\mathrm{RLC}}(\boldsymbol{\varphi})
\end{aligned}
$$

where the time required for transmitting the $N_{u}$ information bits of each RLC-PDU is given by $T_{\mathrm{RLC}}(\boldsymbol{\varphi})=\frac{N_{u} T_{\mathrm{B}}}{r \sum_{(n, q) \in \mathcal{C}} m^{(n, q)}}$, $T_{\mathrm{B}}$ being the OFDM symbol duration. The EGP metric can then be computed as the ratio between the size $N_{p}$ of the data payload and the expected transmission time $\mathcal{T} \triangleq \mathcal{T}_{\mathrm{s}}(\boldsymbol{\varphi}, \boldsymbol{p}) \lambda_{\mathrm{s}}(\boldsymbol{\varphi}, \boldsymbol{p})+$ $\mathcal{T}_{\mathrm{f}}(\boldsymbol{\varphi})\left[1-\lambda_{\mathrm{s}}(\boldsymbol{\varphi}, \boldsymbol{p})\right]$ irrespective of whether the delivery attempt is successful or not. Upon dividing by the overall OFDM bandwidth $\frac{N}{T_{\mathrm{B}}}$, we can eventually get the expression for the normalized EGP as

$$
\zeta(\boldsymbol{\varphi}, \boldsymbol{p})=\frac{N_{p}}{N} \cdot \frac{T_{\mathrm{B}}}{\mathcal{T}_{\mathrm{s}}(\boldsymbol{\varphi}, \boldsymbol{p}) \lambda_{\mathrm{s}}(\boldsymbol{\varphi}, \boldsymbol{p})+\mathcal{T}_{\mathrm{f}}(\boldsymbol{\varphi})\left[1-\lambda_{\mathrm{s}}(\boldsymbol{\varphi}, \boldsymbol{p})\right]} .
$$


Now, since our goal is to guarantee the reliable delivery of the packet at the receiver, it is reasonable to assume the retry limit $R \gg 1$, so that the probability of a successful packet delivery $\lambda_{\mathrm{s}}(\boldsymbol{\varphi}, \boldsymbol{p})$ tends to one, and as a consequence, the average transmission time approximates to $\mathcal{T} \simeq \mathcal{T}_{\mathrm{S}}(\boldsymbol{\varphi}, \boldsymbol{p}) \simeq \frac{T_{\mathrm{RLC}}(\boldsymbol{\varphi})}{\left\{1-\operatorname{PER}_{\varphi}\left[\gamma_{\kappa \mathrm{ESM}}(\boldsymbol{\varphi}, \boldsymbol{p})\right]\right\}}$. The result we end up can then be expressed in the following.

Proposition 2: Under the assumptions of: 1) TM $\varphi$ and power coefficients $\boldsymbol{p}$ independent of the retransmission index; and 2) maximum number of retransmission $R$ attempts adequately large, the normalized EGP can be written as

$$
\zeta(\boldsymbol{\varphi}, \boldsymbol{p})=\zeta_{0} r \sum_{(n, q) \in \mathcal{C}} m^{(n, q)}\left\{1-\operatorname{PER}_{\varphi}\left[\gamma_{\kappa \mathrm{ESM}}(\boldsymbol{\varphi}, \boldsymbol{p})\right]\right\}
$$

where $\zeta_{0} \triangleq \frac{N_{p}}{\left(N N_{u}\right)}$.

Proof: Plugging (9)-(11) into (12) and making $R$ sufficiently large, after some algebra (13) can be easily obtained.

A few concluding remarks are now in order.

1) Selecting the OFDM signal bandwidth as normalization factor, the EGP function can be read as a spectral efficiency metric, in the sense of the data rate conveyed by error-free packet transmitted over a given spectral interval.

2) The EGP function is a monotonically increasing function of the effective $\operatorname{SNR} \gamma_{\kappa \mathrm{ESM}}(\boldsymbol{\varphi}, \boldsymbol{p})$.

3) The choice of different transmission strategies, i.e., $\varphi$ and $\boldsymbol{p}$, affects the EGP metric. The stronger the TM mode, the longer the average transmission time will be, but more likely the delivery will succeed within the retry limit. This fact actually opens the way to the problem of optimizing the EGP metric over $\boldsymbol{\varphi}$ and $\boldsymbol{p}$ that will be the main focus of the next section.

\section{Link Resource AdAptation Optimization Problem}

The task performed by a LRA scheme is that of optimally assigning the available radio resources so that we can get the best link performance for any given channel conditions. The adaptive cross-layer LRA strategy we propose here follows this route in accordance with the $\kappa \mathrm{ESM}$-based LPE framework developed in Section III-B. The idea consists in optimizing the EGP metric in the domain of the PA $\boldsymbol{p}$ and the TM $\boldsymbol{\varphi}$, subject to the constraints on the transmitted power and the finite set $\Phi$ of allowable TMs. Stated formally, our constrained LRA OP is posed as

$$
\left\{\begin{array}{ll}
\left(\boldsymbol{\varphi}_{\mathrm{o}}, \boldsymbol{p}_{\mathrm{o}}\right)=\underset{\boldsymbol{\varphi}, \boldsymbol{p}}{\arg \max }\{\zeta(\boldsymbol{\varphi}, \boldsymbol{p})\} \\
\text { s.t. } \quad \mathbf{1}_{C}^{T} \boldsymbol{p} \leq P \\
\text { s.t. } \quad \boldsymbol{\varphi} \in \Phi
\end{array} .\right.
$$

This is a typical mixed integer OP, where some of the variables are integer while the others are continuous. Given the specific structure of the objective function $\zeta(\boldsymbol{\varphi}, \boldsymbol{p}),(14)$ will be solved by simply dividing it into two steps: first, the optimal power allocation vector $\boldsymbol{p}_{\mathrm{o}}(\boldsymbol{\varphi})$ is derived as a function of the generic $\operatorname{TM} \varphi$, and then, the best TM is selected so that the EGP metric takes its maximum value.

\section{A. Power Allocation Optimization Problem}

The remark mentioned in the above point 2) suggests that the problem of optimizing the EGP metric for a fixed TM turns into the equivalent one of searching for the optimal power allocation $\boldsymbol{p}_{\mathrm{o}}$ that maximizes the $\kappa \mathrm{ESM}$ effective SNR (8). Toward this end, considering that $\psi^{(n, q)}(1)>\psi^{(n, q)}(\mu)$ for $\mu>1$, let us limit the summation in (7) to $\mu=1$ for simplicity, or equivalently, drop the terms relevant to the symbols in the complementary subset at distance greater than $d_{\min }^{(n, q)}$. Then, with

$$
\alpha^{(n, q)} \triangleq \frac{\psi^{(n, q)}(1)}{2^{m^{(n, q)}-1} \cdot \sum_{(i, j) \in \mathcal{C}} m^{(i, j)}}
$$

and $\rho^{(n, q)}$ so that

$$
\frac{1}{\rho^{(n, q)}} \triangleq \frac{\gamma^{(n, q)}}{p^{(n, q)}} \cdot \frac{\left[d_{\min }^{(n, q)}\right]^{2}}{4}
$$

$-\gamma_{\kappa \mathrm{ESM}}$ is conveniently approximated by the function

$$
F(\boldsymbol{p}) \triangleq \log \sum_{(n, q) \in \mathcal{C}} \alpha^{(n, q)} e^{-p^{(n, q)} / \rho^{(n, q)}}
$$

and the PA problem, which will be addressed as "goodput-oriented power allocation" or GOPA for short, can be formalized as

$$
\left\{\begin{array}{l}
\boldsymbol{p}_{\text {o }}=\underset{\boldsymbol{p}}{\arg \min }\{F(\boldsymbol{p})\} \\
\text { s.t. } \quad \mathbf{1}_{C}^{T} \boldsymbol{p} \leq P
\end{array} .\right.
$$

Two propositions can be of help on the way to solve (18). The first one concerns the convexity property of the objective function.

Proposition 3: The real-valued function $F(\boldsymbol{p})$ is strictly convex within the domain $\mathcal{P} \triangleq\left\{p^{(n, q)} \in \mathbb{R}^{+} \mid 1 \leq n \leq N, 1 \leq\right.$ $q \leq M\}$.

Proof: See Appendix A.

Consequently, the GOPA problem fits in the class of convex OPs. Moreover, we can obtain a condition on the components of the gradient of the objective function at the optimal solution, as stated in the following proposition.

Proposition 4: The optimal $\boldsymbol{p}_{\mathrm{o}}$ that solves (18) satisfies

$$
\left.\frac{\partial F(\boldsymbol{p})}{\partial p^{(n, q)}}\right|_{\boldsymbol{p}=\boldsymbol{p}_{\mathrm{o}}} \geq\left.\frac{1}{P} \sum_{(i, j) \in \mathcal{C}} p_{\circ}^{(i, j)} \frac{\partial F(\boldsymbol{p})}{\partial p^{(i, j)}}\right|_{\boldsymbol{p}=\boldsymbol{p}_{\mathrm{o}}}, \quad(n, q) \in \mathcal{C}
$$

with strict equality if $p_{\mathrm{o}}^{(n, q)}>0 \forall(n, q) \in \mathcal{C}$.

Proof: See Appendix B.

Hence, upon exploiting the optimal condition (19), a simple algorithm to derive the optimal solution for the GOPA problem can be derived. For the sake of readability, the algorithm is outlined in Appendix C. Here, we just remark that the optimal solution can be pursued by distributing power among subchannels through a sequence of locally optimal choices: first, allocate power on the subchannel 1 so that the optimal condition of 
Proposition 4 is verified for subchannel 1 and 2 only; then, put power on the subchannels 1 and 2 with equal increments so that Proposition 4 is verified for subchannel 1, 2, and 3 only; go on till the the power constraint is met with strict equality. It can be proved that this rather simple iterative method allows to spend all the available power over the subchannels in a way that solves the OP (18), as stated in the sequel. In fact, upon exploiting (19) a closed-form solution of the constrained OP (18) can be obtained as formalized in the following proposition.

Proposition 5: The optimal $\boldsymbol{p}_{\mathrm{o}}$ solving the GOPA problem has components given by

$$
p_{\mathrm{o}}^{(n, q)}=\rho^{(n, q)}\left[\frac{P-\sum_{(i, j) \in \mathcal{C}} \rho^{(i, j)} \log \frac{\alpha^{(i, j)} \rho^{(n, q)}}{\rho^{(i, j)} \alpha^{(n, q)}}}{\sum_{(i, j) \in \mathcal{C}} \rho^{(i, j)}}\right]^{+},(n, q) \in \mathcal{C}
$$

where $[x]^{+}$equals $x$ if $x>0$ and 0 otherwise.

Proof: See Appendix D.

A few remarks are now in order.

1) Dealing with power coefficients, the values $p_{\mathrm{o}}^{(n, q)}$ must be all nonnegative, which explains the use of the operator $[\cdot]^{+}$. Whenever some of them turn out to be negative, the relevant subchannels are simultaneously switched off, their indexes are removed from $\mathcal{C}$, and (20) is applied again over the resulting subset of $\mathcal{C}$.

2) Assuming $p_{\mathrm{o}}^{(n, q)}>0, \forall(n, q) \in \mathcal{C}$, it is straightforward to verify that

$$
\left.\nabla F(\boldsymbol{p})\right|_{\boldsymbol{p}=\boldsymbol{p}_{\mathrm{o}}}=-\frac{1}{e^{F}\left(\boldsymbol{p}_{\mathrm{o}}\right)+\varepsilon} \cdot \mathbf{1}_{C}
$$

where $\varepsilon \triangleq \frac{1}{\sum_{(i, j) \in \mathcal{C}} \rho^{(i, j)}}\left[P-\sum_{(i, j) \in \mathcal{C}} \rho^{(i, j)} \log \frac{\alpha^{(i, j)}}{\rho^{i, j)}}\right]$, meaning that the gradient of the objective function $F(\boldsymbol{p})$ at the optimal point $\boldsymbol{p}_{\mathrm{o}}$ has equal components in accordance with Proposition 4.

3) The key feature of the optimal solution formalized by Proposition 5 resides in a simple closed-form expression of great convenience in that it depends only on the coefficients $\left\{\rho^{(n, q)}\right\}_{(n, q) \in \mathcal{C}}$ and $\left\{\alpha^{(n, q)}\right\}_{(n, q) \in \mathcal{C}}$.

4) The solution (20) to the GOPA problem can be interpreted in the light of a waterfilling-like representation

$$
\begin{cases}\frac{p_{\mathrm{o}}^{(n, q)}}{\rho^{(n, q)}}=\vartheta-\log \frac{\rho^{(n, q)}}{\alpha^{(n, q)}}, & \log \frac{\rho^{(n, q)}}{\alpha^{(n, q)}}<\vartheta \\ \frac{p_{\mathrm{o}}^{(n, q)}}{\rho^{(n, q)}}=0, & \log \frac{\rho^{(n, q)}}{\alpha^{(n, q)}}>\vartheta\end{cases}
$$

where, due to (16) the ratio $\frac{p_{\circ}^{(n, q)}}{\rho^{(n, q)}}$ is proportional to the SNR level on the $(n, q)$-subchannel after power allocation is performed, whereas $\vartheta \triangleq \frac{P+\sum_{(i, j) \in \mathcal{C}} \rho^{(i, j)} \log \frac{\rho^{(i, j)}}{\alpha^{(i, j)}}}{\sum_{(i, j) \in \mathcal{C}} \rho^{(i, j)}}$ plays the role of the water level.

\section{B. Transmission Mode Optimization Problem}

When the optimal power distribution $\boldsymbol{p}_{\mathrm{o}}(\boldsymbol{\varphi})$ is available as a function of the adopted TM $\varphi$, the constrained LRA OP (14) turns into that of searching the best combination over the set $\Phi$ of the allowable TMs that maximizes the EGP metric $\zeta(\boldsymbol{\varphi}, \boldsymbol{p})$ given by (13), or more formally

$$
\left\{\begin{array}{l}
\boldsymbol{\varphi}_{\mathrm{o}}=\underset{\boldsymbol{\varphi}}{\arg \max }\left\{\zeta\left[\boldsymbol{\varphi}, \boldsymbol{p}_{\mathrm{o}}(\boldsymbol{\varphi})\right]\right\} \\
\text { s.t. } \quad \boldsymbol{\varphi} \in \Phi
\end{array} .\right.
$$

Now, without loss of generality assume that the TM optimization is carried out for a given MIMO setup, although that limitation can be easily dropped by switching among the supported schemes throughout the allocation procedure (as performed while obtaining the results discussed in Section V). Then, we adopt uniform bit allocation (UBA), that is to say, the number of bits $m^{(n, q)}$ assigned to the subchannels is kept equal to $m, \forall(n, q) \in \mathcal{C}$. We remark here that also this restriction can be circumvented resorting to the bit loading scheme proposed in [23] which looks for the optimal set of $m^{(n, q)}$ under given power levels among subchannels. In view of the $\kappa \mathrm{ESM}$ features, the optimization of the GP metric is performed through a greedy-based iterative algorithm which considerably reduces the computational complexity skipping the combinatorial nature of the problem. Hence, the problem of joint bit and power allocation can be split (although in a suboptimal way) into two subproblems each of which is optimally solved at affordable complexity. The joint solution can then be derived resorting to the coordinated ascent method [24]. Thus, (23) can be reformulated as

$$
\left\{\begin{array}{l}
\left(m_{\mathrm{o}}, r_{\mathrm{o}}\right)=\underset{m, r}{\arg \max }\{\bar{\zeta}(m, r)\} \\
\text { s.t. } \quad(m, r) \in \Phi
\end{array}\right.
$$

where the EGP metric to be optimized simplifies to

$$
\bar{\zeta}(m, r)=\bar{\zeta}_{0} r m\left\{1-\operatorname{PER}_{r}\left[\gamma_{\kappa \mathrm{ESM}}(m)\right]\right\}
$$

$\bar{\zeta}_{0} \triangleq \zeta_{0} C, r$ is the coding rate, and $\gamma_{\kappa \mathrm{ESM}}$ is a function of the modulation order $m$ only. The particular shape of (25) helps on solving (24) in a simple way. The idea is to increment $m$ up to the largest value $m_{\max }$ depending on the allowed modulation orders, and for each of them select the optimal $r$ that maximizes the EGP, as defined by the next proposition.

Proposition 6: The $\kappa$ ESM-based LRA strategy solving (24) consists of the following steps:

1) Initialize $m=0, \bar{\zeta}_{\max }=0$;

2) Increment $m$ by 2 ;

3) Compute $\gamma_{\kappa \mathrm{ESM}}(m)$;

4) Select $r^{\prime}=\arg \max _{r}\{\bar{\zeta}(m, r)\}$;

5) If $\bar{\zeta}\left(m, r^{\prime}\right) \geq \bar{\zeta}_{\max }$ and $m \leq m_{\max }$ then

6) Set $m_{\mathrm{o}}=m, r_{\mathrm{o}}=r^{\prime}, \bar{\zeta}_{\max }=\bar{\zeta}\left(m, r^{\prime}\right)$;

7) Go to Step 2);

8) Else, the algorithm finishes returning $m_{\mathrm{o}}$ and $r_{\mathrm{o}}$.

\section{Simulation Results}

In this section, the effectiveness of the proposed LRA method is numerically tested for realistic wireless link scenarios. First, 
the accuracy of the $\kappa \mathrm{ESM}$ link performance model is quantified and compared with conventional ESM-type schemes. Then, the proposed $\kappa$ ESM-based LRA strategy relying on the EGP objective function is evaluated in terms of the actual GP performance for a MIMO BIC-OFDM system, taking as performance benchmark the GP metric that can be obtained by nonadaptive transmissions.

\section{A. System Setup}

Simulation runs will be carried out focusing on two different MIMO BIC-OFDM schemes, designated as Profile A and Profile $B$, respectively. Both of them occupy a $20-\mathrm{MHz}$ RF bandwidth and employ RLC-PDUs each consisting of $N_{p}=1024$ payload binary symbols preceded by the CRC section of length $N_{\text {CRC }}=32$ used by a stop-and-wait ARQ error handling mechanism with 8 logical channels. They differ from each other essentially for the channel propagation model and the type of coding scheme, one using convolutional and the other turbo codes, as outlined in the sequel.

1) Profile A: Profile A employs $N=64$ subcarriers with 4-, 16- and 64-QAM as modulation format, cyclic prefix of length $N_{\mathrm{CP}}=16$, and signalling interval equal to $T=50 \mathrm{~ns}$. Channel encoding is based on a 64-state convolutional code with mother code rate $r=\frac{1}{2}$ and free distance $d_{f}=10$, properly punctured to allow the four rates $1 / 2,2 / 3,3 / 4$, and $5 / 6$, while the propagation model is based on a typical 6-tap MIMO channel with maximum delay spread of $0.6 \mu \mathrm{s}$, each tap being modelled as i.i.d. zero-mean circularly symmetric complex Gaussian RV. We consider the configurations equipped by $N_{\mathrm{T}}$ transmit and $N_{\mathrm{R}}$ receive antennas, with $N_{\mathrm{T}}=N_{\mathrm{R}}=1,2$, or 4 , based on: 1) single transmit and receive antenna (SISO configuration); 2) orthogonal ST block (Alamouti) code (OSTBC) with receiver maximum ratio combining (MRC) [4], or spatial multiplexing (SM) with linear minimum mean square error (MMSE) detector [6], both of them under UBA and UPA, assuming that the $\kappa$ ESM effective SNR be available at the transmitter through a low-rate feedback channel (as depicted in Fig. 1); and 3) SVD-based precoding in the form of the linear unitary precoding (LUP) scheme [7], with UBA and the optimal GOPA or UPA, under the assumption that the transmitter has ideal knowledge of the CSI.

2) Profile B: Profile B employs $N=1320$ active subcarriers, obtained through a 2048-points FFT, with 4-, 16-, and 64-QAM as modulation format, cyclic prefix of length $N_{\mathrm{CP}}=160$, signalling interval equal to $T=50 \mathrm{~ns}$, and SISO antenna configuration only. Channel encoding consists in a 8-state parallel concatenated convolutional turbo code scheme with mother code rate $r=\frac{1}{3}$ and punctured rates $2 / 5,1 / 2,4 / 7$, $2 / 3,3 / 4,4 / 5$, and $6 / 7$, while the propagation channel is modeled as ITU Pedestrian B.

\section{B. Accuracy of the $\kappa E S M$ Performance Model}

The accuracy of the $\kappa \mathrm{ESM}$ performance prediction model is compared to that of the EESM and MIESM conventional methods. This is done by evaluating the complementary cumulative density function (CCDF) of the absolute value of the effective SNR error, which, for the generic channel realization $\boldsymbol{\gamma}_{i}$ $\left(1 \leq i \leq N_{\beta}\right)$ of the subcarrier SNR vector $\boldsymbol{\gamma}$, is defined as

$\varepsilon_{\gamma}\left(\boldsymbol{\gamma}_{i}, \beta, \mathrm{PER}_{i}\right) \triangleq 10 \log _{10} \gamma_{\mathrm{eff}, \beta}\left(\boldsymbol{\gamma}_{i}\right)-10 \log _{10} \gamma_{\mathrm{AWGN}}\left(\mathrm{PER}_{i}\right)$.

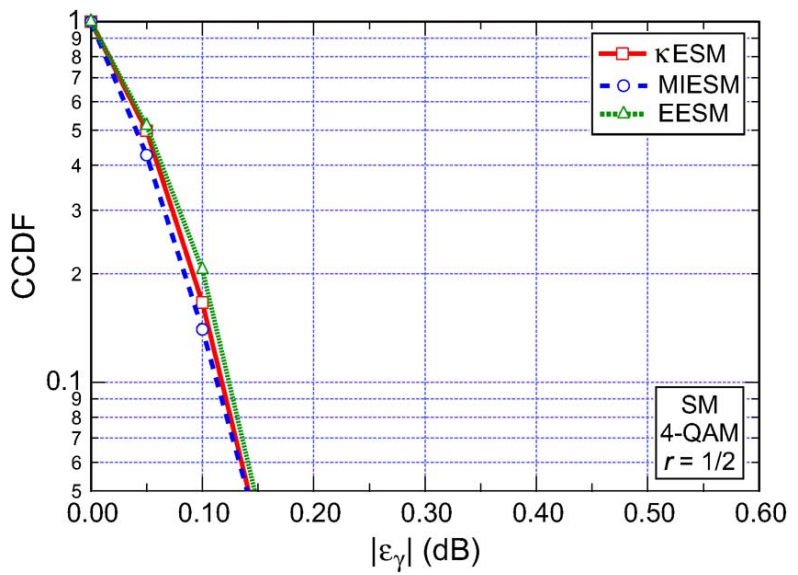

Fig. 2. CCDF of the effective SNR absolute error for SM with 4-QAM and coding rate $r=\frac{1}{2}$ (profile A).

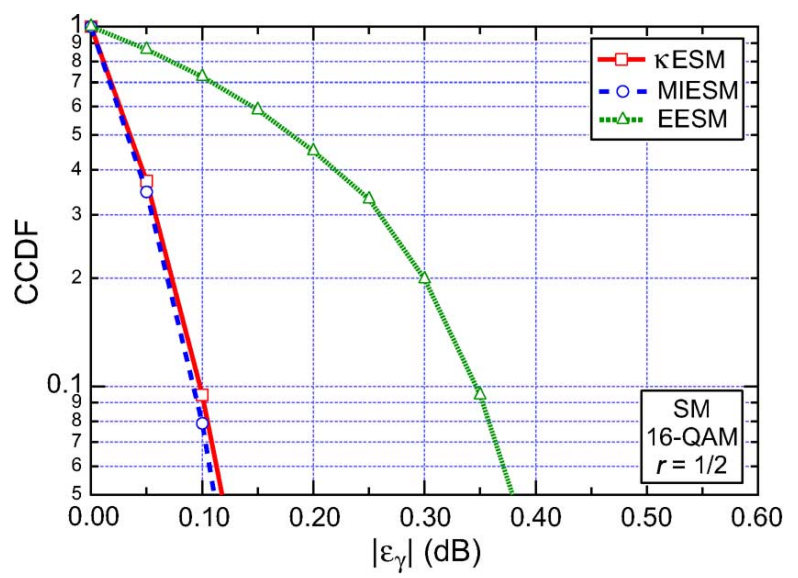

Fig. 3. CCDF of the effective SNR absolute error for SM with 16-QAM and coding rate $r=\frac{1}{2}$ (profile A).

In (26), $\gamma_{\text {eff }, \beta}\left(\boldsymbol{\gamma}_{i}\right)$ is the effective SNR, depending on the SNR values $\gamma_{i}$ measured on the receiver subchannels for the $i$ th channel realization, while $\mathrm{PER}_{i}$ is the PER level corresponding to $\gamma_{i}$ as obtained from link level simulations. Additionally, each ESM method can be equipped with an optimum tuning factor $\beta_{\mathrm{opt}}$ resulting from the least-square fitting

$$
\beta_{\mathrm{opt}}=\underset{\tilde{\beta}}{\arg \min }\left\{\sum_{i=1}^{N_{\beta}}\left|\varepsilon_{\gamma}\left(\boldsymbol{\gamma}_{i}, \tilde{\beta}, \mathrm{PER}_{i}\right)\right|^{2}\right\} .
$$

The optimization of the ESM tuning factors has been carried out for a total number of independent channel realizations $N_{\beta}=$ 100 and for $\mathrm{PER}_{i} \in[0.01,0.95]$.

Figs. 2-4 show the CCDF for 4-, 16-, and 64-QAM, respectively, assuming coding rate $r=\frac{1}{2}$ and $2 \times 2$ SM multiantenna scheme. The better accuracy offered by the $\kappa \mathrm{ESM}$ over the EESM is apparent especially for higher modulation orders. Such an advantage can be promptly explained if one keeps in mind the structure of the $\kappa \mathrm{ESM}$ as composed of two separate models, one for modulation and one for coding, where the former takes specifically into account the modulation format adopted on each subchannel, thus boosting estimate accuracy.

Indeed, in Fig. 4, which refers to a 64-QAM, for a CCDF level of 0.1 the $\kappa \mathrm{ESM}$ offers an absolute error of $0.17 \mathrm{~dB}$, whereas for 


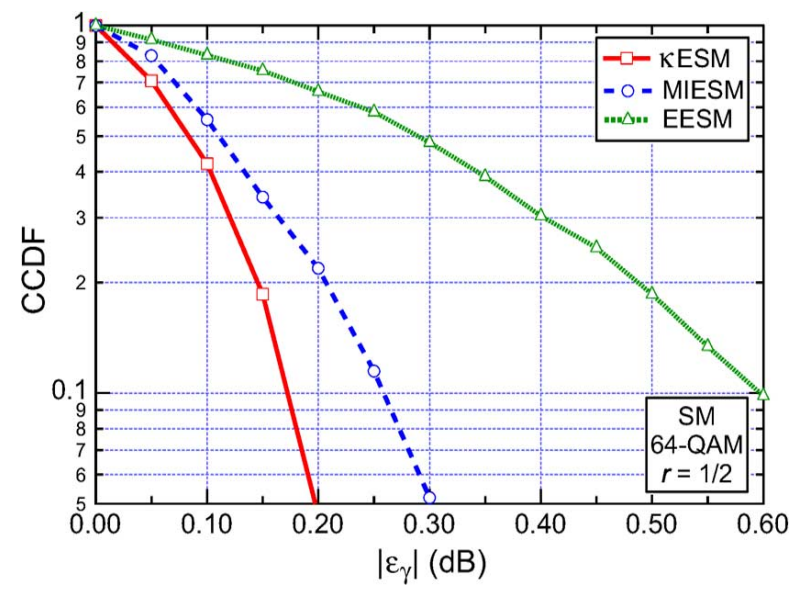

Fig. 4. CCDF of the effective SNR absolute error for SM with 64-QAM and coding rate $r=\frac{1}{2}$ (profile A).

the EESM this turns into around $0.6 \mathrm{~dB}$. On the other side, the gap between the $\kappa \mathrm{ESM}$ and the MIESM is substantially lower, say less than $0.1 \mathrm{~dB}$. Further, it can be derived that the $\kappa \mathrm{ESM}$ guarantees the same slope of the AWGN reference curve whilst this does not hold for the EESM where a "cross point" occurs. This drawback means that, if the EESM were used in combination with an LRA strategy, the selection of the best TM for a given channel condition would not be reliable as based on a biased prediction of the PER link performance. On the other side, the $\kappa \mathrm{ESM}$ enables a PER prediction performance which is very similar to that provided by the MIESM method, but at the price that the mapping function of the latter is neither in closed-form nor convex.

\section{Goodput Performance of the $\kappa E S M$-Based LRA Strategy}

The effectiveness of the $\kappa$ ESM-based LRA strategy is first tested by quantifying the actual GP performance (averaged over $10^{4}$ independent channel realizations) as a function of the $\frac{E_{s}}{N_{0}}$ ratio, under the assumption that at the transmitter side only the effective SNR $\gamma_{\kappa \mathrm{ESM}}$ is available, and UBA is applied together with UPA. Figs. 5 and 6 refers to a SISO link with profiles A and $\mathrm{B}$, respectively, for the adaptive case based on $\kappa \mathrm{ESM}$ (solid line), and all the static TMs (dotted lines with different marks). Note that each modulation format is referenced by a different mark (circles for 4-QAM, squares for 16-QAM and triangles for 64-QAM) with the lower curve corresponding to the lower coding rate. The results obtained for the setups based on both convolutional (Fig. 5, profile A) and turbo codes (Fig. 6, profile B) indicate that the LRA scheme, even assuming simple UPA, effectively offers a gain up to 2 and $3 \mathrm{~dB}$, respectively, if compared with the best-performing static TM.

The above results obtained for the SISO configuration are substantially confirmed in Fig. 7 for the $2 \times 2$ SM scheme with MMSE detector. It has to be remarked that such a setup represents a demanding test for the proposed LRA method, since in this case the SM receiver performance is significantly affected by interstream interference. Nevertheless, the adaptive $\kappa \mathrm{ESM}$-based LRA (solid line) reveals to be quite robust and keeps the capability of selecting the best-performing TM (dotted lines with marks), even reaching up to $1 \mathrm{~dB}$ gain.

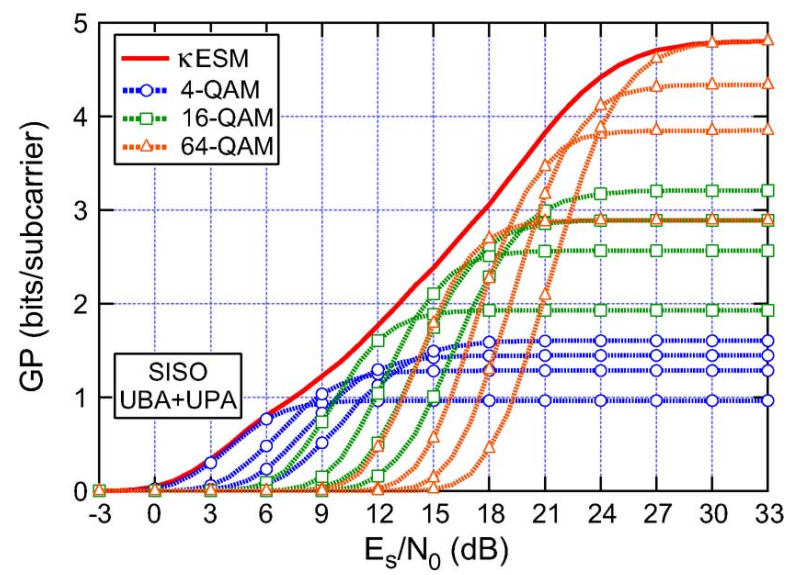

Fig. 5. GP performance of the adaptive $\kappa \mathrm{ESM}$ and static modes for SISO (profile A).

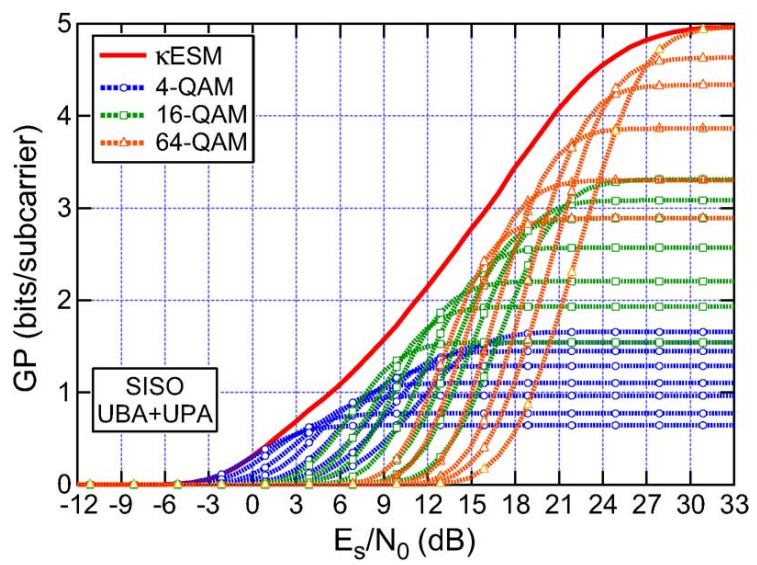

Fig. 6. GP performance of the adaptive $\kappa \mathrm{ESM}$ and static modes for SISO (profile B).

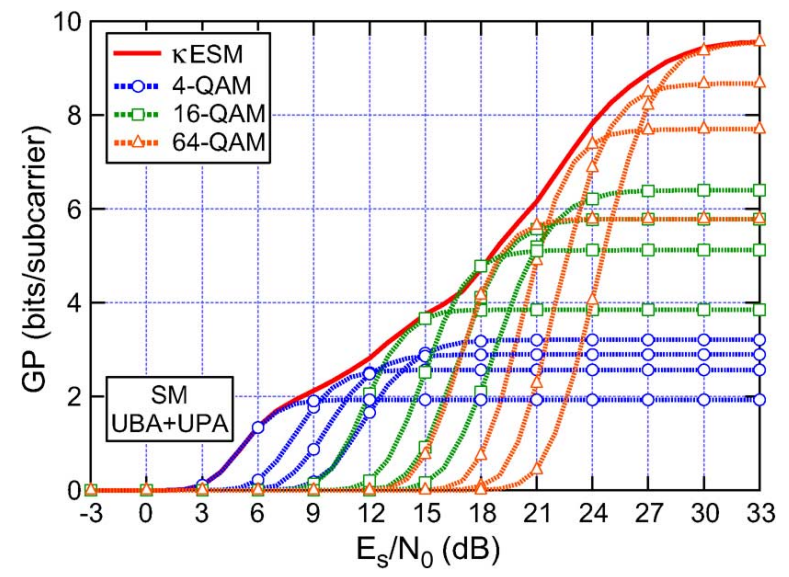

Fig. 7. GP performance of the adaptive $\kappa \mathrm{ESM}$ and static modes for SM (profile A).

According to the $\kappa \mathrm{ESM}$ framework illustrated in Section III-B, the scalar $\gamma_{\kappa \text { ESM }}$ works as a simple yet accurate indicator at the transmitter side of the current link quality. As such, it enables not only the optimization of the modulation order and coding rate but also the adaptive selection of the best multiantenna scheme. This interesting feature is verified in Fig. 8 that thus extends the results presented 


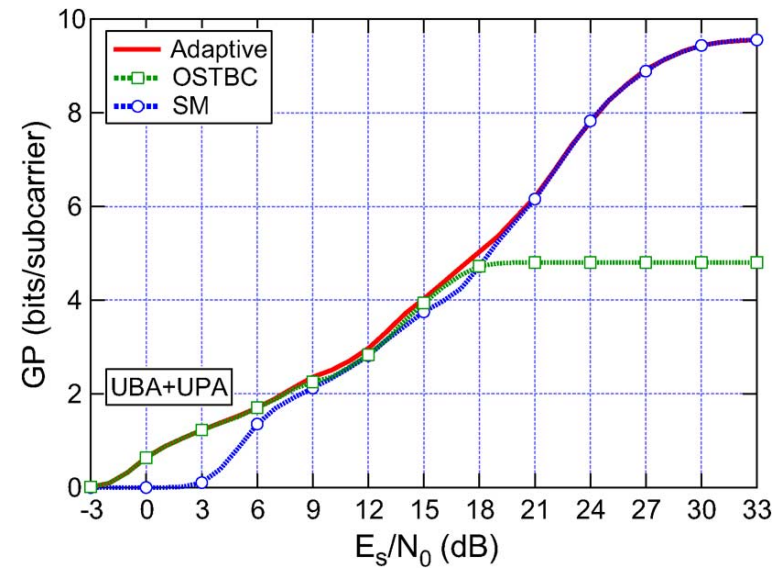

Fig. 8. GP performance of the adaptive $\kappa \mathrm{ESM}$ versus fixed OSTBC and SM (profile A).

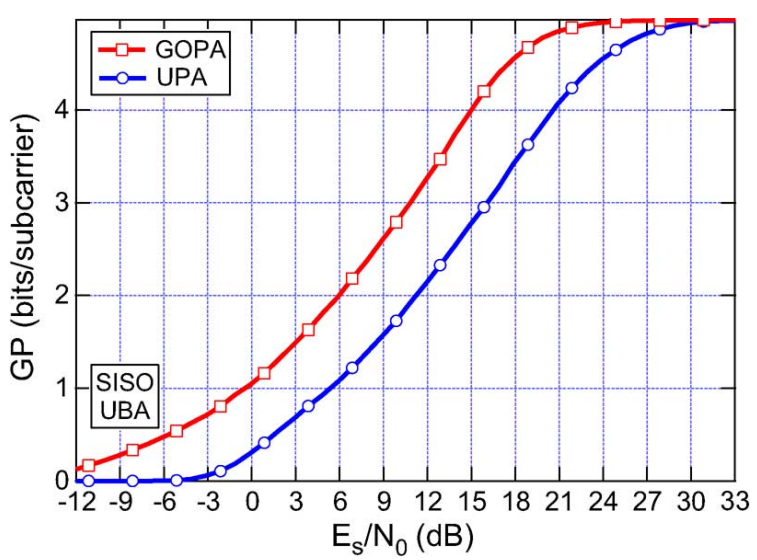

Fig. 9. GP performance of the adaptive $\kappa$ ESM with GOPA or UPA for SISO (profile B).

in Fig. 7, by comparing the GP performance with optimal modulation and coding rate for: 1) fixed $2 \times 2$ OSTBC (dotted line with squares); 2) fixed SM (dotted line with circles); and 3) adaptive multiantenna switching (solid line) based on the optimal selection at each packet transmission of OSTBC or SM scheme. It is apparent that the adaptive curve almost overlaps with the "envelope" of the fixed OSTBC or SM curves, which means that the adaptive scheme gets the best GP performance for each $\frac{E_{s}}{N_{0}}$ value of interest.

Let us now address the GP link performance improvement that can be gained when the transmitter has ideal knowledge of the current channel conditions, i.e., the vector $\gamma$, and UBA is adopted together with either UPA or the optimal GOPA as power distribution schemes. At first, in Fig. 9 we compare the UPA against the GOPA when the SISO configuration is considered for the profile $\mathrm{B}$. The optimal power distribution given by the GOPA algorithm (solid line with squares) allows a substantial gain over the UPA scheme (solid line with circles) ranging from 5 to around $8 \mathrm{~dB}$ depending on the value of the $\frac{E_{s}}{N_{0}}$ ratio. This is evident, indeed, especially for the low-SNR regime, where the GOPA exploits at best its capability of optimally distributing power among the available subcarriers, and even if required, switching off those that experience very low SNR. Then, in Fig. 10, a $4 \times 4$ multiantenna setup for the profile $\mathrm{A}$ is taken into account, which allows to decompose the MIMO channel

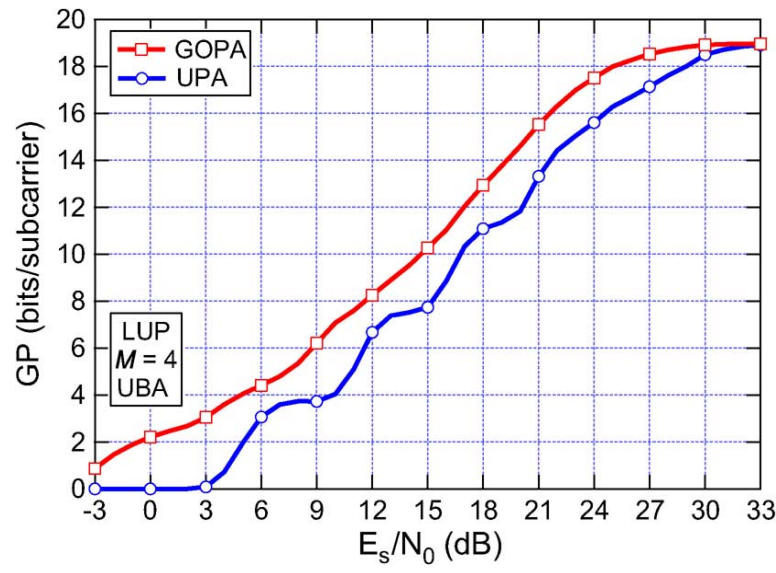

Fig. 10. GP performance of the adaptive $\kappa$ ESM with GOPA or UPA for LUP with $M=4$ (profile A).

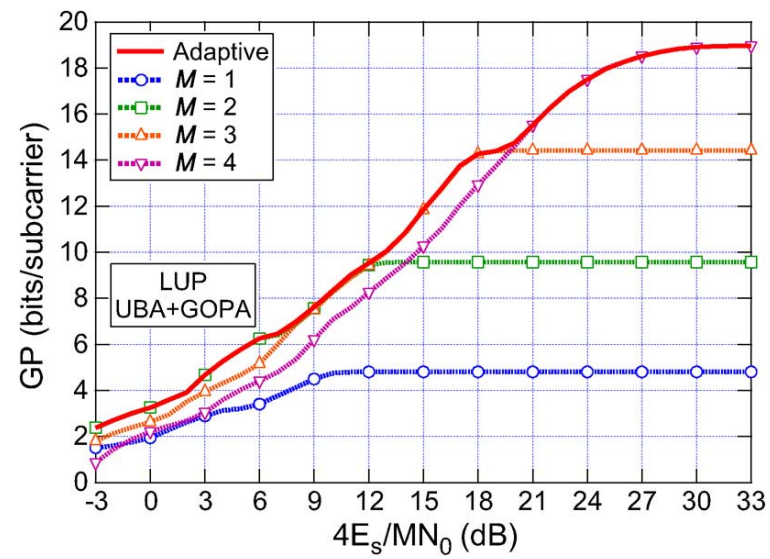

Fig. 11. GP performance of the adaptive and fixed $M \kappa \mathrm{ESM}$ for LUP with GOPA (profile A).

into fixed $M=4$ parallel streams through the SVD-based precoding/decoding (LUP scheme). The performance improvement exhibited by the GOPA over the UPA is similar to that shown in the SISO case (see Fig. 9), but ranges from 2 to more than $6 \mathrm{~dB}$.

Besides optimally choosing the power distribution across the subcarriers, the modulation order and coding rate, the LRA algorithm has also the potential of selecting the number of active spatial streams which turn out to be available when the LUP scheme is adopted. As apparent from Fig. 11, the adaptive $\kappa$ ESM-based method (solid line) offers a GP metric that almost coincides with that of the best-performing MIMO configuration (dotted lines with marks) with fixed number of streams $M, 1 \leq M \leq 4$. Hence, despite the complexity of the overall multiparametric OP, the proposed LRA scheme is effective in performing the optimal selection of all the transmission system parameters which yield the best possible GP for any SNR value.

\section{CONCLUDING REMARKS}

We have addressed the design of a novel cross-layer LRA scheme for soft-decoded MIMO BIC-OFDM transmissions employing ARQ. First, the $\kappa$ ESM link performance evaluation model has been analytically derived in a simple closed-form and advantageously compared with conventional methods. Numerical results demonstrate that it outperforms EESM, especially 
for high modulation orders, and provides performance similar to that of MIESM, while offering a reduced complexity. Then, an LRA procedure based on the $\kappa$ ESM framework has been formalized with the aim of maximizing the goodput metric over the allowable link resources, such as power distribution among the active subchannels, modulation order, coding rate and multiantenna setup. The performance tests show that the link parameters can be effectively matched to the instantaneous channel conditions. In the case of no CSI available at the transmitter, i.e., when only a low-rate scalar feedback is permissible, the proposed LRA strategy yields a gain with respect to the best static transmission mode around $2-3 \mathrm{~dB}$ for SISO and $1 \mathrm{~dB}$ for $2 \times 2$ MIMO configurations, respectively. On the other side, in the case of perfect CSI at the transmitter, the optimal power distribution provided by the GOPA algorithm allows a substantial goodput improvement over the uniform power allocation scheme, ranging from 2 to more than $6 \mathrm{~dB}$.

\section{APPENDIX}

\section{A. Proof of Proposition 3}

It can be found from (17) that the function $F(\boldsymbol{p})=\log \left(\boldsymbol{\rho}^{T} \boldsymbol{\xi}\right)$ is twice differentiable in the domain $\mathcal{P}$ with gradient $\nabla F(\boldsymbol{p})=$ $-\frac{1}{\boldsymbol{\rho}^{T} \boldsymbol{\xi}} \boldsymbol{\xi}$ and Hessian $\nabla^{2} F(\boldsymbol{p})=\frac{1}{\left(\boldsymbol{\rho}^{T} \boldsymbol{\xi}\right)^{2}}\left[\left(\boldsymbol{\rho}^{T} \boldsymbol{\xi}\right) \operatorname{diag}(\boldsymbol{\xi}) \boldsymbol{\eta}-\boldsymbol{\xi} \boldsymbol{\xi}^{T}\right]$, where $\boldsymbol{\xi} \triangleq\left[\frac{\alpha_{1}}{\rho_{1}} e^{-\frac{p_{1}}{\rho_{1}}}, \cdots, \frac{\alpha_{P}}{\rho_{P}} e^{-\frac{p_{P}}{\rho_{P}}}\right]^{T}, \boldsymbol{\eta} \triangleq\left[\frac{1}{\rho_{1}}, \cdots, \frac{1}{\rho_{P}}\right]^{T}$, and $\boldsymbol{\rho} \triangleq\left[\rho_{1}, \cdots, \rho_{P}\right]^{T}, P$ being the number of active subchannels, $p_{(n-1) M+q} \triangleq[\boldsymbol{p}]_{(n-1) M+q}=p^{(n, q)}, \alpha_{(n-1) M+q} \triangleq$ $\alpha^{(n, q)}$, and $\rho_{(n-1) M+q} \triangleq \rho^{(n, q)}$, with $n, q \in \mathcal{C}$. Then, consider $\boldsymbol{v}^{T} \nabla^{2} F(\boldsymbol{p}) \boldsymbol{v}=\frac{1}{\left(\boldsymbol{\rho}^{T} \boldsymbol{\xi}\right)^{2}}\left[\left(\boldsymbol{\rho}^{T} \boldsymbol{\xi}\right) \boldsymbol{v}^{T} \operatorname{diag}(\boldsymbol{\xi}) \boldsymbol{\eta} \boldsymbol{v}-\boldsymbol{v}^{T} \boldsymbol{\xi} \boldsymbol{\xi}^{T} \boldsymbol{v}\right], \forall \boldsymbol{v} \in$ $\mathcal{P}$. Upon dropping an immaterial proportional factor, from the Cauchy-Schwarz inequality applied to the vectors $f \triangleq$ $\left[\sqrt{\xi_{1} \rho_{1}}, \cdots, \sqrt{\xi_{P} \rho_{P}}\right]^{T}$ and $\boldsymbol{g} \triangleq\left[v_{1} \sqrt{\frac{\xi_{1}}{\rho_{1}}}, \cdots, v_{P} \sqrt{\frac{\xi_{P}}{\rho_{P}}}\right]^{T}$, we obtain $\boldsymbol{v}^{T} \nabla^{2} F(\boldsymbol{p}) \boldsymbol{v}=\left(\boldsymbol{f}^{T} \boldsymbol{f}\right)\left(\boldsymbol{g}^{T} \boldsymbol{g}\right)-\left(\boldsymbol{f}^{T} \boldsymbol{g}\right)>0$. This proves that the Hessian of $F(\boldsymbol{p})$ is positive definite, and consequently, the function is strictly convex.

\section{B. Proof of Proposition 4}

According to the convex optimization theory framework [25], in the minimization of a convex objective function subject to a convex constraint set, there exists an unique $\boldsymbol{p}_{\mathrm{o}}$ such that $F\left(\boldsymbol{p}_{\mathrm{o}}\right)<F(\boldsymbol{p})$, or in equivalent words, whenever a local optimal solution is found, then it is the global solution as well. The directional derivative of $F(\boldsymbol{p})$ at $\boldsymbol{p}_{\mathrm{o}}$ in the direction of any other $\boldsymbol{p}$ results therefore as

$$
\nabla F\left(\boldsymbol{p}_{\mathrm{o}}\right)^{T}\left(\boldsymbol{p}-\boldsymbol{p}_{\mathrm{o}}\right)=\left.\sum_{(n, q) \in \mathcal{C}}\left[p^{(n, q)}-p_{\mathrm{o}}^{(n, q)}\right] \frac{\partial F(\boldsymbol{p})}{\partial p^{(n, q)}}\right|_{\boldsymbol{p}=\boldsymbol{p}_{\mathrm{o}}} \geq 0
$$

where the strict equality holds if $p^{(n, q)}>0, \forall(n, q) \in \mathcal{C}$. By exploiting the affine property on $\boldsymbol{p}$, the condition (28) can be evaluated on the extreme points of the set, namely $p^{\left(n^{\prime}: q^{\prime}\right)}=P$, and $p^{(n, q)}=0 \forall(n, q) \neq\left(n^{\prime}, q^{\prime}\right)$, thus obtaining the claimed expression.

\section{GOPA Algorithm}

As initial step: $i$ ) set the power allocation vector $\boldsymbol{p}=\mathbf{0}$; ii) take the gradient of the objective function $F(\boldsymbol{p})$ at $\boldsymbol{p}=\mathbf{0}$ and evaluate its components as

$$
\left.\frac{\partial F(\boldsymbol{p})}{\partial p^{(n, q)}}\right|_{\boldsymbol{p}=\mathbf{0}}=-\frac{1}{\sum_{(i, j) \in \mathcal{C}} \alpha^{(i, j)}} \cdot \frac{\alpha^{(n, q)}}{\rho^{(n, q)}}, \quad(n, q) \in \mathcal{C}
$$

iii) sort the absolute values of (29) in a decreasing order; $i v$ ) enumerate the ordered subchannels through the index $j, 1 \leq j \leq$ $C ; v)$ assign to the $j$ th subchannel $p^{(j)} \triangleq p^{\left(n_{j}, q_{j}\right)}, \alpha_{j} \triangleq \alpha^{\left(n_{j}, q_{j}\right)}$ and $\left.\rho_{j} \triangleq \rho^{\left(n_{j}, q_{j}\right)} ; v\right)$ rename the sequence of power coefficients as $\boldsymbol{p}=\left[p^{(1)}, p^{(2)}, \cdots, p^{(C)}\right]^{T}$. Then, we consider $J$ steps, being $J \leq C$ the number of active subchannels, in each of which the power is progressively incremented so that the optimal condition of gradient with equal components formulated in Proposition 4 is met. Note that we admit implicitly that all the components of the optimal solution we are searching for are strictly greater than zero. Going into details, the power vector $\boldsymbol{p}_{j}$ allocated at step $j, 1<j \leq J$, can be written as $\boldsymbol{p}_{j}=\boldsymbol{p}_{j-1}+\delta \boldsymbol{p}_{j}$, where $\delta \boldsymbol{p}_{j} \triangleq\left[p_{j}^{(1)}, p_{j}^{(2)}, \cdots, p_{j}^{(j)}, 0, \cdots, 0\right]^{T}$. Hence, the $J$ steps of interest can be detailed as follows.

- Step 1) Set $\boldsymbol{p}_{1}=\left[p_{1}^{(1)}, 0, \cdots, 0\right]^{T}$ and impose the condition

$$
\left.\frac{\partial F(\boldsymbol{p})}{\partial p^{(1)}}\right|_{\boldsymbol{p}=\boldsymbol{p}_{1}}=\left.\frac{\partial F(\boldsymbol{p})}{\partial p^{(2)}}\right|_{\boldsymbol{p}=\boldsymbol{p}_{1}}
$$

thus obtaining $p_{1}^{(1)}=\rho_{1}\left[\log \frac{\alpha_{1}}{\rho_{1}}-\log \frac{\alpha_{2}}{\rho_{2}}\right]$.

- Step 2) Set $\boldsymbol{p}_{2}=\left[p_{1}^{(1)}+p_{2}^{(1)}, p_{2}^{(2)}, 0, \cdots, 0\right]^{T}$ and impose

$$
\left.\frac{\partial F(\boldsymbol{p})}{\partial p^{(1)}}\right|_{\boldsymbol{p}=\boldsymbol{p}_{2}}=\left.\frac{\partial F(\boldsymbol{p})}{\partial p^{(2)}}\right|_{\boldsymbol{p}=\boldsymbol{p}_{2}}=\left.\frac{\partial F(\boldsymbol{p})}{\partial p^{(3)}}\right|_{\boldsymbol{p}=\boldsymbol{p}_{2}} .
$$

From the second equality, we get $p_{2}^{(2)}=$ $\rho_{2}\left[\log \frac{\alpha_{2}}{\rho_{2}}-\log \frac{\alpha_{3}}{\rho_{3}}\right]$, whereas from the first one $p_{1}^{(1)}+p_{2}^{(1)}=\rho_{1}\left[\log \frac{\alpha_{1}}{\rho_{1}}-\log \frac{\alpha_{3}}{\rho_{3}}\right]$ and $\frac{p_{2}^{(1)}}{\rho_{1}}=\frac{p_{2}^{(2)}}{\rho_{2}}$.

- Step j) Set $\boldsymbol{p}_{j} \triangleq\left[\sum_{m=1}^{j} p_{m}^{(1)}, \cdots, \sum_{m=j}^{j} p_{m}^{(j)}, 0, \cdots, 0\right]^{T}$ and impose

$\left.\frac{\partial F(\boldsymbol{p})}{\partial p^{(1)}}\right|_{\boldsymbol{p}=\boldsymbol{p}_{j}}=\left.\cdots \frac{\partial F(\boldsymbol{p})}{\partial p^{(j)}}\right|_{\boldsymbol{p}=\boldsymbol{p}_{j}}=\left.\frac{\partial F(\boldsymbol{p})}{\partial p^{(j+1)}}\right|_{\boldsymbol{p}=\boldsymbol{p}_{j}}$.

Exploiting the $j$ th equality, we get $p_{j}^{(j)}=$ $\rho_{j}\left[\log \frac{\alpha_{j}}{\rho_{j}}-\log \frac{\alpha_{j+1}}{\rho_{j+1}}\right]$ from which it can be proved by induction that

$$
\sum_{m=n}^{j} p_{m}^{(n)}=\rho_{n}\left[\log \frac{\alpha_{n}}{\rho_{n}}-\log \frac{\alpha_{j+1}}{\rho_{j+1}}\right]
$$

with $1 \leq n \leq j$, and $\frac{p_{j}^{(1)}}{\rho_{1}}=\frac{p_{j}^{(2)}}{\rho_{2}}=\cdots=\frac{p_{j}^{(j)}}{\rho_{j}}$. Repeat the generic step $j$ until

$$
\sum_{n=1}^{j} \sum_{m=n}^{j} p_{m}^{(n)} \geq P .
$$


- Step $J$ ) Consider the step $j=J$ such that

$$
\sum_{n=1}^{J} \sum_{m=n}^{J} p_{m}^{(n)} \geq P
$$

then recompute the last increment $p_{J}^{(n)}$ with $1 \leq n \leq J$, imposing

$$
\sum_{n=1}^{J} p_{J}^{(n)}+\sum_{n=1}^{J-1} \sum_{m=n}^{J-1} p_{m}^{(n)}=P
$$

together with the local optimal condition (19).

Finally, return the power allocation $\boldsymbol{p}_{J}$ and stop the algorithm.

\section{Proof of Proposition 5}

Because of its decreasing monotonicity, the objective function is minimized when the power constraint in (18) is met with strict equality. Accordingly, upon plugging (33) into the constraint

$$
\sum_{n=1}^{C} \sum_{m=n}^{C} p_{m}^{(n)}=P
$$

the expression (20) is eventually found.

\section{REFERENCES}

[1] D. Astély, E. Dahlman, P. Frenger, R. Ludwig, M. Meyer, S. Parkvall, P. Skillermark, and N. Wiberg, "A future radio-access framework," IEEE J. Sel. Areas Commun., vol. 24, no. 3, pp. 693-706, Mar. 2006.

[2] J. A. C. Bingham, "Multicarrier modulation for data transmission: An idea whose time has come," IEEE Commun. Mag., vol. 28, no. 5, pp. 5-14, May 1990.

[3] G. Caire, G. Taricco, and E. Biglieri, "Bit-interleaved coded modulation," IEEE Trans. Inf. Theory, vol. 44, no. 5, pp. 927-946, May 1998.

[4] S. M. Alamouti, "A simple transmit diversity technique for wireless communication," IEEE J. Sel. Areas Commun., vol. 16, no. 8, pp. 1451-1458, Oct. 1998.

[5] V. Tarokh, H. Jafarkhani, and A. R. Calderbank, "Space-time block codes from orthogonal designs," IEEE Trans. Inf. Theory, vol. 45, no. 5, pp. 1456-1467, Jul. 1999.

[6] A. J. Paulraj, D. A. Gore, R. U. Nabar, and H. Bölcskei, "An overview of MIMO communications-A key to gigabit wireless," Proc. IEEE, vol. 92, no. 2, pp. 198-218, Feb. 2004.

[7] M. Vu and A. J. Paulraj, "MIMO wireless linear precoding," IEEE Signal Process. Mag., vol. 24, no. 5, pp. 86-105, Sep. 2007.

[8] S. Lin, D. Costello, and M. J. Miller, "Automatic-repeat-request errorcontrol schemes," IEEE Commun. Mag., vol. 22, no. 12, pp. 5-17, Dec. 1984.

[9] G. Klang et al., Identification of Radio-Link Technologies IST-2003507581, WINNER Project, D2.1 Public Deliverable.

[10] Q. Liu, S. Zhou, and G. B. Giannakis, "Cross-layer combining of adaptive modulation and coding with truncated ARQ over wireless link," IEEE Trans. Wireless Commun., vol. 3, no. 5, pp. 1746-1755, Sep. 2004.

[11] A. Lozano, A. Tulino, and S. Verdu, "Optimum power allocation for parallel Gaussian channels with arbitrary input distribution," IEEE Trans. Inf. Theory, vol. 52, no. 7, pp. 3033-3051, Jul. 2006.

[12] K.-B. Song, A. Ekbal, S. T. Chung, and J. M. Cioffi, "Adaptive Modulation and Coding (AMC) for Bit-Interleaved Coded OFDM (BIC-OFDM)," IEEE Trans. Wireless Commun., vol. 5, no. 7, pp. 1685-1694, Jul. 2006.

[13] E. Akay, E. Sengul, and E. Ayanoglu, "Bit interleaved coded multiple beamforming," IEEE Trans. Commun., vol. 55, no. 9, pp. 1802-1811, Sep. 2007.

[14] D. Qiao, S. Choi, and K. G. Shin, "Goodput analysis and link adaptation for IEEE 802.11 a wireless LANs," IEEE Trans. Mobile Comput., vol. 52, no. 4, pp. 278-292, Oct.-Dec. 2002.
[15] B. Devillers, J. Louveaux, and L. Vandendorpe, "Bit and power allocation for goodput optimization in coded parallel subchannel with ARQ," IEEE Trans. Signal Process., vol. 56, no. 8, pp. 3652-3661, Aug. 2008.

[16] S. Nanda and K. M. Rege, "Frame error rates for convolutional codes on fading channels and the concept of effective $\frac{E_{b}}{N}$," IEEE Trans. Veh. Technol., vol. 47, no. 4, pp. 1245-1250, Nov. 1998.

[17] E. Tuomaala and H. Wang, "Effective SINR approach of link to system mapping in OFDM/multi-carrier mobile network," in Proc. 2nd Int. Conf. Mobile Technol., Appl. Syst., Nov. 15-17, 2005, 5 pp..

[18] Y. W. Blankenship, P. J. Sartori, B. K. Classon, V. Desai, and K. L. Baum, "Link error prediction methods for multicarrier systems," in Proc. IEEE 60th Veh. Technol.Conf. 2004 (VTC 2004-Fall), Sep. 2004, vol. 6, pp. 4175-4179.

[19] L. Wan, S. Tsai, and M. Almgren, "A fading-insensitive performance metric for a unified link quality model," in Proc. IEEE Wireless Commun. Netw. Conf. 2006 (WCNC 2006), Apr. 2006, vol. 4, pp. 2110-2114.

[20] I. Stupia, F. Giannetti, V. Lottici, and L. Vandendorpe, "A novel exponential link error prediction method for OFDM systems," Multi-Carrier Syst. Solutions 2009 (MC-SS 2009), pp. 291-300, May 2009.

[21] A. Martinez, A. Guillèn i Fàbregas, and G. Caire, "Error probability analysis of bit-interleaved coded modulation," IEEE Trans. Inf. Theory, vol. 52, no. 1, pp. 262-271, Jan. 2006.

[22] M. R. McKay, I. B. Collings, A. Forenza, and R. W. Heath, Jr., "Multiplexing/beamforming switching for coded MIMO in spatially correlated channels based on closed-form BER approximations," IEEE Trans. Veh. Technol., vol. 56, no. 5, pp. 2555-2567, Sep. 2007.

[23] I. Stupia, F. Giannetti, V. Lottici, R. Andreotti, L. Vandendorpe, and A. D'Andrea, "A greedy algorithm for goodput-oriented AMC in turbocoded OFDM," in Proc. Future Network and Mobile Summit, 2010 (FUNEMS 2010), Jun. 2010.

[24] D. P. Bertsekas, Nonlinear Programming, 2nd ed. Singapore: Singapore: Athena Scientific, 2003.

[25] S. Boyd and L. Vandenberghe, Convex Optimization. Cambridge, U.K.: Cambridge Univ. Press, 2004.

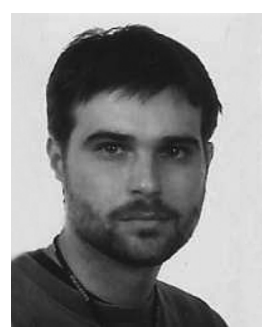

Ivan Stupia received the M.Sc. degree in telecommunications engineering and the Ph.D. degree in information engineering from the University of Pisa, Pisa, Italy, in 2004 and 2009, respectively.

Since 2011, he has been with the Communications and Remote Sensing Laboratory of UCL. From 2009 to 2011 , he held a postdoctoral position in the Department of Information Engineering at the University of Pisa, and during 2007, he was a visiting Ph.D. student at the University of Louvain, Louvain-la-Neuve, Belgium. His expertise and general interests span the areas of wireless communications and signal processing with special emphasis on adaptive modulation and coding, resource allocation, cross-layer design, and nonlinear distortion issues. His current research interests include the application of convex optimization and game theory to signal processing, and to the design of efficient cognitive radio transceivers.

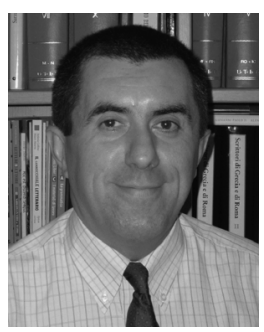

Vincenzo Lottici received the Dr.Ing. degree (cum laude) and the BTA in electrical engineering both from the University of Pisa, Pisa, Italy, in 1985 and 1986, respectively.

From 1987 to 1993 , he was engaged in the research of sonar digital signal processing algorithms. Since 1993, he has been with the Department of Information Engineering, University of Pisa, where he is currently an Assistant Professor of Communication Systems. He participated in several international and national research projects, and specifically, from 2005 he has been taking an active role in the standardization activity promoted by ETSI (European Telecommunications Standards Institute) of the physical layer of the mobile radio system TETRA release2. He frequently served as technical program committee (TPC) member for major IEEE conferences in wireless communications and signal processing, such WCNC2012, ICUWB2011, ICC2011, WCNC2011, ICASSP2010, PIMRC2010, WCNC2010, CIP2010, Globecom2009, SPAWC2009, EUSIPCO2006, Globecom2006. His research interests include the broad area 
of signal processing for communications, with emphasis on synchronization, dynamic resource allocation, cognitive radio, and compressive sensing.

Dr. Lottici received the Best Paper Award in 2006 for the work A Theoretical Framework for Soft Information Based Synchronization in Iterative (Turbo) Receivers, (EURASIP Journal on Wireless Communications and Networking, April 2005), by the EU-funded project Network of Excellence in Wireless Communications (NEWCOM).

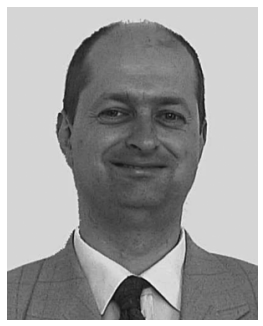

Filippo Giannetti was born in Pontedera, Italy, in 1964. He received the Doctor Engineer (cum laude) and the Research Doctor degrees in electronic engineering from the University of Pisa, Pisa, Italy, in 1989 and from the University of Padova, Padova, Italy, in 1993, respectively.

In 1992, he spent a research period at the European Space Agency Research and Technology Centre (ESA/ESTEC), Noordwijk, The Netherlands, where he was engaged in several activities in the field of digital satellite communications. From 1993 to 1998, he has been a Research Scientist at the Department of Information Engineering, University of Pisa, where he is currently an Associate Professor of Telecommunications. His main research interests are in the field of wireless communications, with special emphasis on digital modem design, digital signal processing algorithms, wideband transmission techniques, and resource allocation in cognitive and cooperative networks.

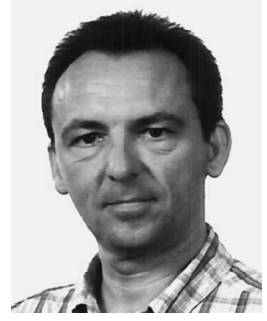

Luc Vandendorpe (M'93-SM'99-F'06) was born in Mouscron, Belgium, in 1962. He received the B.Eng. degree (summa cum laude) and the Ph.D. degree from the Université Catholique de Louvain (UCL), Louvain-la-Neuve, Belgium, in 1985 and 1991, respectively.

Since 1985, he has been with the Communications and Remote Sensing Laboratory of UCL, where he first worked in the field of bit rate reduction techniques for video coding. In 1992, he was a Visiting Scientist and Research Fellow at the Telecommunications and Traffic Control Systems Group, Delft Technical University, Delft, The Netherlands, where he worked on spread spectrum techniques for personal communications systems. From October 1992 to August 1997, he was Senior Research Associate of the Belgian NSF at UCL. He is currently a Full Professor and head of the Institute for Information and Communication Technologies, Electronics and Applied Mathematics. His current research interest include digital communication systems and, more precisely, resource allocation for OFDM(A)-based multicell systems, MIMO and distributed MIMO, sensor networks, turbo-based communications systems, physical layer security, and UWB based positioning.

Dr. Vandendorpe was corecipient of the 1990 Biennal Alcatel-Bell Award from the Belgian NSF for a contribution in the field of image coding. In 2000, he was corecipient (with J. Louveaux and F. Deryck) of the Biennal Siemens Award from the Belgian NSF for a contribution about filter-bank-based multicarrier transmission. In 2004, he was cowinner (with J. Czyz) of the Face Authentication Competition, FAC 2004. He is or has been TPC member for numerous IEEE conferences (VTC Fall, Globecom Communications Theory Symposium, SPAWC, ICC) and for the Turbo Symposium. He was Cotechnical Chair (with P. Duhamel) for the IEEE ICASSP 2006. He was an Editor for Synchronization and Equalization of the IEEE TRANSACTIONS ON COMMUNICATIONS between 2000 and 2002, Associate Editor of the IEEE TRANSACTIONS ON WIRELESS COMMUNICATIONS between 2003 and 2005, and Associate Editor of the IEEE TRANSACTIONS ON SignAl Processing between 2004 and 2006. He was Chair of the IEEE Benelux joint chapter on Communications and Vehicular Technology between 1999 and 2003. He was an elected member of the Signal Processing for Communications committee between 2000 and 2005 and between 2009 and 2011, and an elected member of the Sensor Array and Multichannel Signal Processing committee of the Signal Processing Society between 2006 and 2008. He is the Editor-in-Chief for the EURASIP Journal on Wireless Communications and Networking. 